\title{
Filigrane
}

Écoutes psychanalytiques

\section{Ce qui cherche à se figurer : du dicible et du visible dans le cadre de l'analyse et en psychothérapie à médiation}

\section{Josée Leclerc}

Volume 27, numéro 2, 2018

La consultation psychanalytique aujourd'hui, entre héritages et remaniements

URI : https://id.erudit.org/iderudit/1055755ar

DOI : https://doi.org/10.7202/1055755ar

Aller au sommaire du numéro

Éditeur(s)

Revue Santé mentale au Québec

ISSN

1192-1412 (imprimé)

1911-4656 (numérique)

Découvrir la revue

Citer cet article

Leclerc, J. (2018). Ce qui cherche à se figurer : du dicible et du visible dans le cadre de l'analyse et en psychothérapie à médiation. Filigrane, 27(2), 125-138. https://doi.org/10.7202/1055755ar
Résumé de l'article

Comment comprendre la puissance de l'image, sa capacité à signifier ce qui nous " regarde " (Didi-Huberman, 1992) ? Comment la psychothérapie à médiation artistique peut-elle répondre aux visées cliniques qui guident les aménagements du cadre requis avec certains patients pour qui la cure-type n'est pas indiquée ? Il s'agira de prendre en considération certains points de convergence propres à la méthode psychanalytique telle qu'elle se déploie dans la situation analysante et en psychothérapie psychanalytique médiatisée, propos centré sur le pouvoir de figuration du mot et de l'image, sur

l'associativité qui en découle et sur la disposition du psychothérapeute à saisir ce qui se présente dans le récit du patient, qu'il soit langage verbal ou visuel. Le cas d'une patiente souffrant de dépression majeure servira d'étayage afin de montrer comment la mise en oeuvre de dispositifs d'objectivation permet le travail de symbolisation d'expériences psychiques primaires jusque-là non métabolisées. 


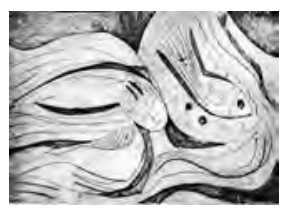

\title{
Ce qui cherche à se figurer: du dicible et du visible dans le cadre de l'analyse et en psychothérapie à médiation
}

\author{
Josée Leclerc
}

\begin{abstract}
Résumé: Comment comprendre la puissance de l'image, sa capacité à signifier ce qui nous «regarde»(Didi-Huberman, 1992)? Comment la psychothérapie à médiation artistique peut-elle répondre aux visées cliniques qui guident les aménagements du cadre requis avec certains patients pour qui la cure-type n'est pas indiquée? Il s'agira de prendre en considération certains points de convergence propres à la méthode psychanalytique telle qu'elle se déploie dans la situation analysante et en psychothérapie psychanalytique médiatisée, propos centré sur le pouvoir de figuration du mot et de l'image, sur l'associativité qui en découle et sur la disposition du psychothérapeute à saisir ce qui se présente dans le récit du patient, qu'il soit langage verbal ou visuel. Le cas d'une patiente souffrant de dépression majeure servira d'étayage afin de montrer comment la mise en œuvre de dispositifs d'objectivation permet le travail de symbolisation d'expériences psychiques primaires jusque-là non métabolisées.
\end{abstract}

Mots clés: situation analysante; psychothérapie psychanalytique médiatisée; figuration; associativité; disposition psychique.

\begin{abstract}
How is one to understand the power of images, their capacity to signify what "regards" us (Didi-Huberman, 1992)? How can art-mediated psychotherapy respond to the clinical aims guiding the rearrangements of the setting required for certain patients for whom classical analysis is not indicated? Taking into consideration certain points of convergence inherent in the psychoanalytic method, such as it is used in the analysing situation and in mediated psychoanalytic psychotherapy, my remarks centre on the figurative power of words and images and on the psychotherapist's readiness to receive and acknowledge what presents itself in the patient's narrative in verbal or visual form. The case of a patient suffering from a major depression will serve as substantiation in order to show how the implementation of objectivating techniques facilitates the symbolisation work of primary psychic experiences not metabolised up to that point.
\end{abstract}

Keywords: analysing situation; mediated psychoanalytic psychotherapy; figurative power of words and images; associativity; psychic disposition. 
L

'image est là devant nous déposée. Elle vient de naître des mains de la patiente. L'image intime au silence, pour qui veut bien la laisser parler. La patiente y est disposée. Du mode triadique image-patiente-psychothérapeute qui constitue le cadre de la psychothérapie psychanalytique médiatisée ${ }^{1}$, la place est pour l'heure concédée à la première. Nos regards s'y posent. La patiente sait que si c'est elle qui a créé l'image, c'est l'image qui contient les secrets qu'elle y a mis à son insu, guidée par la matière imageante, selon les tracés et modelés qui se sont esquissés, dans un rapport d'analogie ou d'opposition. La vie dans l'image importe moins que la vie de l'image (Schaverien, 1992). Dans cet espace potentiel, le figuratif s'ouvre peu à peu au figural qui le constitue. La représentation cède sa place dominante; ses contours s'estompent et laissent apparaitre non pas l'informe mais le moins de forme. Configurations secondaires, détails, fragments, accidents se manifestent et revendiquent l'espace. D'incongrus ou de banals, apparemment sans histoire, ils deviennent prégnants, parfois nimbés d'une lumière particulière lorsque, se détachant du fond, soudainement ils se présentent au regard. Espace d'accueil à l'image, suivant le lien - contingent ou essentiel, demandera Pontalis (1988, p. 280) - entre image visuelle et image psychique. Mais peut-être plus encore, espace d'ouverture à l'accueil de l'image, par elle initié.

Comment comprendre les pouvoirs de l'image ${ }^{2}$, ses effets d'atteinte, comme je l'ai théorisé ailleurs (Leclerc, 2004, 2012 a, 2012 b), sa capacité à nous dessaisir et à signifier ce qui nous « regarde» (Didi-Huberman, 1992)? Comment la psychothérapie psychanalytique médiatisée peut-elle servir les visées cliniques qui guident aujourd'hui les aménagements du cadre requis, dans certains contextes et avec certains patients, quand la cure-type s'avère impuissante à produire son travail d'introspection et de perlaboration? La médiation artistique est-elle pour autant restreinte à ce seul contexte?

Prendre en considération certains points de convergence dans la méthode pour faire advenir l'inconscient centré sur le traitement des mots et des images permettra d'esquisser des éléments de réponse à ces questions. Cette élaboration métapsychologique sera dirigée non pas sur ce qu'il s'agirait d'entendre dans la parole du patient en analyse ou ce qu'il s'agirait de voir dans les images du patient en psychothérapie psychanalytique médiatisée, mais bien plutôt sur ce qui se donne à voir et à entendre dans la matérialité du signifiant, qu'il se présente sous une forme langagière visuelle ou verbale. Une attention particulière sera, dans ce contexte, accordée à la disposition du psychothérapeute requise par le travail de figuration des contenus 
psychiques que la situation psychothérapique fait émerger. Un exemple clinique issu de ma pratique clinique servira d'étayage à ces théorisations.

\section{Figurations}

Si la substance psychique inconsciente «devient audible à l'analyste c'est, induit Jean-Claude Rolland ${ }^{3}$, par le biais d'images qu'il en construit et dont la production doit obéir, comme Freud l'a clairement suggéré, au principe de l'hallucination» (2006, p. 58). On sera d'abord sensible au devenir audible de la substance psychique dont parle Rolland qui, selon la magnifique formule de F. Gantheret (1989), serait à la fois voilée et révélée dans le discours de l'analysant. Je reviendrai à la disposition du thérapeute que sous-tend l'énoncé de Rolland mais soulignerai ici l'intimité du lien prévalant entre ce que l'entendu donne à voir et ce que le voir donne à entendre. Pour que cela se présente à l'entendement et que l'analyste perçoive ce qui se signale à son écoute flottante, non seulement le mot se sera-t-il détaché de sa signification logique, mais il aura fallu qu'il se fasse voir avec les images, mentales, psychiques, que cet entendu provoque en lui. Vision, fantaisie, rêverie, une fantaiserie, selon le lapsus qui s'est glissé dans mon écriture, qui fait de l'écoutant-regardant un visionnaire, terme cher à Rolland.

Si en psychothérapie psychanalytique médiatisée, il y a présence d'une image matérielle concrète, celle que réalise le patient dans le temps de la séance, on doit convenir avec Jacques Rancière qu'une des opérations principales de l'image de l'art réside dans la mise en jeu d'un « rapport du dicible au visible» (2003, p. 15) que sa production actualise. Motivée quant au but, c'est-à-dire que le cadre même en surdétermine la fonction, l'image en psychothérapie à médiation est autant sujet de l'investigation que sujet à l'investigation. Elle s'inscrit également dans un double rapport, soit du dicible au visible et du visible au dicible: exprimer, dire, montrer quelque chose (dicible), consciemment et inconsciemment, sous une forme picturale ou graphique (visible), adresse au thérapeute chargé de la recevoir; puis procéder à l'exploration associative de ce que l'image, une fois réalisée et accueillie, renvoie, ce qui engage la parole, qu'elle soit mise en mots ou silencieuse.

On reconnaitra à la fois l'arbitraire et la pertinence de cette séquence puisque visible et dicible sont toujours dans « un rapport qui joue en même temps sur leur analogie et sur leur dissemblance» (Rancière, 2003, p. 15). D'où que le mot fasse image et que l'image soit parlante. L'image n'est «pas une exclusivité du visible», pas plus que le mot une exclusivité du dicible. «Il y a du visible qui ne fait pas image, il y a des images qui sont toutes en 
mots. Le visible se laisse disposer en tropes significatifs, la parole déploie une visibilité qui peut être aveuglante» (p. 5). Convenir qu'il y ait du visible dans le dicible et du dicible dans le visible grâce au rapport qui les unit me semble une avenue théorico-clinique féconde afin de penser ce qui de la méthode pour rendre conscient l'inconscient ou, mieux encore, pour faire sens de la réalité psychique du patient, peut concerner aussi bien ce qui s'exprime en mot qu'en image.

Il faut pour cela reconnaitre «la parenté sensorielle de l'expérience visuelle et de l'expérience acoustique» (Rolland, 2006, p. 84); pas n'importe quelle expérience mais bien celle qui induit chez le récepteur ou l'entendant une forme particulière d'éprouvé somatopsychique, un évènement d'atteinte qui cristallise l'attention, saisit la pensée et provoque la survenue d'images psychiques. Ce qui du récit narratif manifeste du patient se présente alors à l'œil ou à l'oreille rend sensible les traces que les mots, et j'ajouterais, les images, auront gardés «de leur participation à l'expérience inconsciente»(Rolland, 2006, p. 84). D'où la qualité hallucinatoire de ces images psychiques, leur puissance figurale résidant dans l'effet de présentation qu'elles induisent, au-delà d'une forme représentative en tant que telle: Darstellung ou mieux Rücksicht auf Darstellbarkeit, prise en considération de la figurabilité, cette notion incontournable de la pensée freudienne (1900) permet de penser l'opération singulière du travail du rêve suivant laquelle les pensées du rêve se présentifient en images principalement visuelles. Au psychothérapeute interprète revient la tâche de prendre en considération ce qui revêt une forme figurale pour se manifester et de reconnaitre que suivre le fil associatif de ces images figurées, substitutives, mène quelque part parce qu'elles viennent de quelque part ${ }^{4}$. Qu'il émane du discours du patient, des images issues de la médiation artistique quand une forme ou tout autre excès de matière imageante produit une effet d'effraction dans le tissu de la représentation manifeste, ou encore de rêves narrés en paroles ou en images, le potentiel d'évocation du mot et de l'image rend ainsi perceptible au psychothérapeute ce qui cherche à se présenter pour être travaillé dans la situation psychothérapique et l'actualisation du transfert: «un travail de vérité qui se porte au-devant d'une vérité en travail» (Pontalis, 1978, p. 37). C'est ce qu'entend Rolland par devenir signifiant.

Pour l'auteur de Avant d'être celui qui parle, les images psychiques que son écoute du discours du patient suscite servent de voie royale, pourrais-je dire, vers la reconnaissance d'analogies qu'abrite le récit du patient à son insu. Il s'agit pour l'analyste d'entendre dans ce qui se dit se redit ou, en 
d'autres termes, d'entendre la redite inconsciente d'un dire conscient, ce qui sera nommé, si je me permets de résumer son propos, à peu près de la manière suivante: Vous parlez de ceci (situation ou évènement présent conscient) et il semble que ça vous soit venu en tête quand vous avez pensé à cela (réminiscence inconsciente). Il y a, entre les deux énoncés, conscient et inconscient, l'existence d'un rapport analogique essentiel. Existence, dis-je, car cela témoigne de la vie de la chose inconsciente, son appartenance aux représentations-choses pré-langagières du registre du processus primaire, son autonomie à se présenter en images visuelles principalement, et sa force d'attraction (Drang). "C'est dans ce mouvement si particulier du retour du refoulé que l'analogie se produit.» (Rolland, 2003, p. 67)

Pour que les configurations auxquelles je faisais référence en ouverture du présent texte se présentent dans l'image créée par le patient en séance, au cour de la substance imageante, et effectuent une brèche dans le refoulement ou un effet d'insight, il aura fallu l'œil visionnaire du psychothérapeute, sa capacité, dans l'attention flottante, à percevoir le signifiant dans l'anodin. Il aura fallu assurément la prise en considération de la figurabilité, tel que décrit ci-dessus, processus de transposition ou de transfert de pensées ou d'expériences inconscientes dans une forme imageante qui rend visible plus qu'elle ne donne à voir, grâce aux images psychiques qui se manifestent à la psyché du psychothérapeute; ou, plus justement, grâce au travail de figuration qui incombe au psychothérapeute suivant une double fonction: abriter dans sa psyché ces images psychiques, figurations principalement visuelles, le temps nécessaire à leur traduction, comme Bion (2005) l'entendait, et figurer le sens qu'elles indiquent de la réalité psychique du patient.

Et il aura fallu, enfin, la parole du psychothérapeute qui attire l'attention du patient et guide son regard: «et ces deux petits traits, en haut à gauche, dans votre image...?» Non pas qu'il connaisse par avance le sens précis de ce qu'il signale. Mais ce qui se démarque de l'ensemble, brèche dans le système représentatif, lorsque mis en relief par une parole ouverte du clinicien - peut-être moins rapprochement analogique dans ce cas que soulignement d'une différence - agit comme une invite au patient qui provoque un arrêt sur l'image et déclenche un processus associatif et noétique.

Si l'attention flottante de l'analyste se porte aux pensées mises en mots dans le récit du patient, aux silences, coupures ou brisures qui le constituent également, elle ne sera nullement indifférente à toute forme d'expression non verbale, corporelle et affective, comme autant d'indicateurs signifiants de la vie psychique du patient. De même, il incombe au clinicien de savoir 
discerner ce qui se dessine dans le dispositif-cadre de la psychothérapie psychanalytique médiatisée et tout autant d'entendre ce qui se signale dans le processus même de mise en forme avec la matière, la manière suivant laquelle le patient procède, s'engage corporellement et affectivement avec la matière, ou se retient. Le processus créatif en dit autant que l'œuvre achevée sur la réalité psychique et affective du patient, voire sur ses relations objectales dont l'objet de médiation peut se faire le révélateur. C’est dire la fonction paradigmatique de l'attention portée à la communication implicite, entre psychés, dans l'entendement et le voir psychiques.

\section{Médiation}

Toute pratique artistique, pour qui s'y engage, instaure un espacetemps qui n'est pas celui de l'orientation ordinaire, participe d'une quête qui excède souvent le champ décisionnel logique et entraine une abdication temporaire du contrôle du moi. Le cadre méthodologique de la psychothérapie psychanalytique médiatisée repose sur l'instauration d'un tel espace, une aire intermédiaire d'expérience dans laquelle la création a lieu, comme Winnicott a su le faire valoir. Ni réalité interne ni réalité externe mais les deux à la fois, au sein de cet espace particulier d'expérience, l'image matérielle, grâce à la fonction transitionnelle de la médiation et celle jouée par le médium malléable ${ }^{5}$, se fait surface d'accueil rendant sensible la permanence du lien prévalant entre ce qui s'exprime dans le récit visuel manifeste du patient et l'empreinte de sa liaison avec l'expérience psychique inconsciente; puissance figurale, il en était question ci-dessus.

À la fois objet matériel objectivement perçu et objet symbolique subjectivement conçu, ni uniquement matériel ni symbolique seulement, le statut paradoxal de l'image issue de la médiation en est un qui ne doit pas être contesté. Les mots de Winnicott (1951) concernant l'objet transitionnel trouvé-créé par l'enfant se distinguent par leur pertinence:

[...] l'on peut dire qu'à l'égard de l'objet transitionnel, il existe une entente entre nous et l'enfant: on ne lui demandera jamais "as-tu eu l'idée de telle chose ou est-ce que cela t'est venu de l'extérieur?» Ce qui compte, c'est qu'on ne s'attend pas à ce que l'enfant prenne position; la question ne doit même pas être formulée. (p. 267)

Pour que la médiation fasse son travail psychothérapique d'élaboration et de perlaboration, le maintien d'une organisation paradoxale s'impose 
assurément: une croyance profonde dans le pouvoir de l'image, son potentiel de figuration et de symbolisation, doit coexister avec la certitude qu'il ne s'agit que d'une image (Weir, 1987). Trop de réalité entrave l'illusion que la création requiert; trop de proximité avec les jeux de l'illusion pourra entrainer une perte des repères du moi, une déprise ou une dépersonnalisation du sujet; nombre d'artistes et de poètes se sont abimés dans l'abysse de la quête.

Or, il y a du visible qui ne fait pas image. Il arrive que l'accès à un espace potentiel d'expérience soit forclos ou sérieusement entravé. C'est le cas de patients présentant des pathologies sévères, états limites, fonctionnement psychotique, décompensation dépressive, par exemple, patients dont la capacité de symbolisation est inexistante ou limitée, contrainte par l'expérience traumatique primaire, l'indifférenciation sujet-objet, l'attachement déficient, l'absence de contenance, de régulation émotionnelle ou d'accordage. L'accès aux jeux métaphoriques de l'illusion créatrice, l'ouverture à l'imprévu des surgissements créateurs et à la libre association ne font alors pas partie des registres d'expériences accessibles. À la non-médiatisation de l'expérience chez ces patients correspond la concrétude de l'expression. Privés de leur pouvoir d'évocation, collés à la réalité, les mots et les images se contentent de la représenter ou de la répéter.

«[...] Un quart des patients inscrits sur la liste d'attente [du centre Favreau], souligne Baldacci, dispose d'une topique psychique bien différenciée permettant le retour sur soi, la confrontation à la règle fondamentale et l'analysabilité des conflits entre le désir et l'interdit.» (Baldacci, 2010, p. 65) Pour les autres patients, ceux, nombreux, qui souffrent de psychopathologies graves, les aménagements du dispositif-cadre s'avèrent un impératif clinique autant qu'éthique. Le recours à des modalités d'objectivation susceptibles de «faire apparaître [le] conflit au dehors " s'impose, de manière à «en permettre une introjection secondaire» (Baldacci, 2010, p. 66) : psychodrame analytique, psychothérapie psychanalytique médiatisée, psychothérapie psychanalytique corporelle, par exemple.

La vignette clinique d'une patiente suivie en psychothérapie psychanalytique médiatisée ${ }^{6}$ présentée ci-après pourra témoigner d'un tel processus d'objectivation et montrer l'utilité de l'expérience contre-transférentielle comme vecteur de connaissance de la réalité psychique de patients qui, comme elle, n'ont pas bénéficié d'un environnement en mesure de métaboliser les expériences primaires vécues et les affects concomitants. Mais dans ce contexte, c'est sans doute l'identification projective qui, en dépit de son exigence, permet au psychothérapeute d'éprouver la teneur émotionnelle et 
affective de la douleur psychique qui les habite et ce, d'autant que la parole chez ces patients est souvent contrainte, perçue comme dangereuse ou encore figée dans le réel.

La patiente saisit le pastel bleu trace rudement sur le papier des lignes horizontales infléchies par une légère courbe une ligne bleue une autre puis une autre encore saisit le pastel rouge trace par-dessus les lignes bleues sans les couvrir entièrement une ligne rouge une autre puis une autre encore ainsi de suite avec la même intensité saisit le pastel noir refait les mêmes gestes les mêmes lignes son mouvement se fait plus lent s'arrête dépose le pastel noir regarde le dessin se met à pleurer doucement de plus en plus abondamment de silencieux ses pleurs deviennent bruyants des sanglots agitent son corps qui hurle sa douleur.

Hormis quelques moments de trêve, le processus créatif et son effet d'affect se rejoueront à peu près de la même manière, à chacune des séances pendant plus d'un an. Chaque dessin créé par la superposition des couches successives renvoie à cette patiente son état et le redouble, intensifie le sentiment d'être dans une impasse et accroit la profonde détresse qu'elle éprouve.

La dépression sévère atteint cette jeune femme, cycliquement depuis quelques années. Elle ne sait pas pourquoi. Bien sûr sa mère était fragile, dépression post-partum après sa naissance et autres épisodes dépressifs majeurs à valence psychotique ayant nécessité des hospitalisations psychiatriques; "mais pas si majeurs que ça ", tient-elle à préciser. Cette fois, pour elle, c'est pire que jamais: sentiment de perdre pied, de s'enfoncer toujours davantage, elle n'en peut plus, a peur de revivre un autre séjour en institut de soins psychiatriques, peur de passer à l'acte mais écarte le suicide, pour le moment, dit-elle, pour ne pas causer de peine à sa mère et la faire souffrir. La relation entre elles est symbiotique. Le père a quitté femme et enfant quand la patiente avait moins d'un an. Elle est l'unique enfant.

Quand elle trace plus qu'elle ne dessine, je suis des yeux le trajet des lignes répétées, les couleurs apposées en succession: bleu-calme, rouge-irritation, noir-dépression, nommera-t-elle. J'entends le mouvement du pastel sec qui marque le papier avec l'énergie du désespoir, sinon elle ne bougerait pas, resterait immobile, le mouvement qui progressivement se modère, ralentit, jusqu'à se faire presque imperceptible; je vois la lenteur d'exécution du noir appliqué de manière hypnotique et absente, le noir du pastel qui recouvre la feuille de son dépôt cendreux tache ses doigts qu'elle frotte et tord compulsivement, puis elle pleure bruyamment sa détresse, à voir son image. Son désespoir m'atteint, me fait questionner la validité du dispositif, 
l'effet de renvoi de ses images provoquant une atteinte narcissique profonde qui, dans l'actualité des séances, la replonge corps et âme dans l'état dépressif, alors que se rejoue compulsivement l'enfermement du retour du même qui provoque le désespoir, ainsi de suite, en boucle et en continu.

Quelques rares tentatives interprétatives, quelques reflets indirectement esquissés-demeurent vains, j'entends dans mes propres paroles l'écho du vide. Je note cependant que la régularité des séances semble contribuer au maintien de la stabilité de son état affectif qui ne s'améliore ni ne s'aggrave.

C'est une chose de savoir qu'il me faut tolérer l'inscription du processus créatif de cette patiente dans la compulsion de répétition, que son traitement graphique a certainement à voir avec une expérience primaire traumatique qui, se rejouant continûment, essaie de se symboliser, et qu'intervenir à ce stade serait contre-indiqué. Mais c'en est parfois une autre que de faire l'épreuve de l'identification projective; tout thérapeute connaît la saisie psychique et affective qui en résulte, le temps que celle-ci se libère et nous délivre. Une fois, tentative afin de permettre, peut-être, une brèche dans la reproduction du même, je l'ai invitée, si elle le souhaitait, à fermer les yeux avant de s'engager dans la production de son image et à les garder fermés ou ouverts, à sa convenance, durant le processus créatif ${ }^{7}$. Ce qu'elle discerne dans le tracé réalisé les yeux mi-clos la désespère: elle voit dans son image des mains, des mains disproportionnées auxquelles elle associe aussitôt ses besoins qui, dit-elle, sont démesurés. Elle a honte de ses besoins, de leur ampleur, et honte de les avoir représentés. Qui plus est, elle dit penser que maintenant que je connais l'étendue de ses besoins, je ne voudrai plus continuer à la suivre en psychothérapie.

Premier signe de l'actualisation du transfert, le fantasme que la séquence décrite ci-dessus rend manifeste le lien psychique prévalant entre la perception de ses besoins affectifs démesurés comme formes honteuses inavouables et la conséquence qu'entraine l'idée qu'ils soient connus par l'autre: que cet autre, et en l'occurrence sa psychothérapeute dans le transfert objectal primaire, l'abandonne. Mais encore, c'est la représentation de ses besoins, leur figuration dans une forme visible symbolique, je le souligne, qui me les donne à voir, opération par moi initiée, qui actualise le fantasme. La suite du processus psychothérapique en révèlera la source mais, bien que le propos ne s'applique pas intégralement au présent contexte, je rappelle ici «la peur d'utiliser le clinicien comme matière à symboliser, dont fait état Roussillon, voire la terreur d'utiliser les humains-soignants pour leur faire subir cette chose innommable d'eux-mêmes [...], avec le cortège de honte et 
de culpabilité que cela implique». Et d'ajouter: «Il faut parfois des années pour que certains patients acceptent de nous utiliser de manière impitoyable» (Roussillon, 2010, p. 29). La réitération du processus - superpositions des lignes en couches successives, pleurs (qui se font moins intenses), désespoir - se poursuivra pendant bien des séances encore.

«Et ces deux petits traits en haut à gauche, dans votre dessin?» Deux petits traits verticaux, peu visibles mais néanmoins présents, émergent de l'agglomérat de lignes horizontales. "Ma mère et moi», associe-t-elle spontanément, puis se met à pleurer violemment. Un souvenir refait surface: elle a quatre ou cinq ans, sa mère tient sa main dans le sienne et repousse les cuticules de ses ongles, un doigt à la fois, dans un geste répétitif, absent, maintes fois recommencé. Je comprendrai qu'elle devra tolérer l'inconfort et le malaise qu'elle ressent et réprimer son envie de retirer sa main: donner sa main à sa mère absentée dans une sorte de psychose blanche (Donnet et Green, 1973), pour qu'elle ne parte pas, ne sombre pas plus avant dans la folie. Je comprendrai également que, vécue dans l'absence de pare-excitation et dans la hantise de la perte et de l'abandon maternels, cette expérience d'effraction corporelle non intégrable psychiquement se reproduira fréquemment. Les deux petits traits émergents dans son image, tels qu'évoqués ci-dessus, se sont avérés surfaces de transfert de l'expérience traumatique inconsciente.

Au lieu du papier Canson qui sert toujours de support à ses dessins, la patiente saisit une mince feuille de papier de soie sur lequel elle reproduit le rituel habituel des tracés avec les pastels secs. Je suis sur le qui-vive craignant, si la fragile feuille de soie devait se déchirer sous la rigidité du médium, qu'une décompensation ne se produise. Il n’y aura pas de déchirure.

Qui-vive, le vocable me reste en tête, se fait entendre autrement dans l'après-coup: qui vive? Ma mère pas moi, pour qu'elle vive? Sorte de pacte sacrificiel suivant lequel ses propres besoins (désirs) sont tus ou tués pour maintenir en vie l'objet maternel, le destin pulsionnel aura pris la forme du retournement en passivité (Freud, 1915), la répression des désirs-besoins leur conférant un dynamisme psychique d'autant plus puissant qu'il doit être réprimé; d'où la nature honteuse et inavouable de ceux-ci, et le rejet fantasmé que leur satisfaction pourrait provoquer ${ }^{8}$.

Selon le sens ancien de la locution interjective qui vive?, il revenait à la sentinelle en poste la tâche de sommer toute personne s'approchant de sa garde de répondre au «qui vive?» qu'elle lui adressait, son devoir étant de parer à toute menace potentielle. Aussi l'interjection formulée était-elle le 
fait de sa vigilance. L'usage fait par ma patiente de la feuille de soie (feuille de soi?) aura induit en moi l'éprouvé (qui-vive) qui dans l'indentification projective m'aura rendue sensible à l'intensité affective, tant de la vigilance déployée envers l'objet maternel, que de la peur d'effondrement éprouvée s'il fallait que le retrait de sa main abandonnée à sa mère ne signe une déchirure définitive.

Une variante se produit: je note qu'en une sorte d'effleurement, la patiente estompe parfois avec ses mains les lignes tracées au pastel. Beaucoup plus tard et pour la première fois, elle inscrit en fin de séance un titre au bas de son dessin: «je veux toucher», n'en dit pas plus. Du traitement maternel subi passivement lors des séances de stimulation des cuticules de ses doigts de main, au désir d'être celle qui touche, qui veut toucher, il semble qu'un renversement psychique s'opère. Les mots du titre sont écrits, ils ne sont pas nommés mais en attente de traduction par la psychothérapeute chargée de les recevoir. C'est une expérience de type trouver-créer qui le permettra.

Sorte de "contretransfert concordant» (Racker, 1963) à son désir de toucher, je découvre dans un magasin de matériel d'artistes visité par hasard, une pâte à modeler blanche, simple à utiliser, douce au toucher, qui n'a pas le caractère régressant de l'argile et durcit à l'air libre. Je place le bloc dans son emballage ciré dans un des casiers du secrétaire qui sert de table de travail dans mon cabinet. Installée à la table, la patiente le voit, s'en saisit, l'ouvre et dit aimer l'odeur de la pâte qui lui rappelle les bricolages effectués chez sa grand-mère quand celle-ci la gardait chez elle entre autres durant les séjours de sa mère en institution psychiatrique. Ces souvenirs sont heureux, elle se sent en sécurité, peut bricoler et jouer. Si le médium est «donné pour utilisation » par le thérapeute, soutient Roussillon, «il doit être approprié et conquis par l'utilisateur» (2010, p. 31).

Depuis lors, la patiente n'utilisera que ce médium, ce qui donnera lieu à la création d'un bestiaire et autres petits objets, suivant un processus inscrit dans une temporalité réglée - elle les crée lors d'une séance et y applique de la couleur lors de la séance suivante quand ils ont séchés -, et selon un cycle oscillatoire d'endommagement et de réparation. Si la fluidité de l'aquarelle permet une expérience réparatrice, il en est tout autrement de l'usage des feutres à pointes fines qu'elle utilise aussi pour tracer des lignes (encore) sur la pâte sèche et rêche de l'objet, comme autant de scarifications. Je suis le témoin passivisé du «mauvais traitement » fait aux objets disposée, cette fois, à le tolérer, le contenir et le figurer de manière à le lui rendre psychiquement envisageable. La malléabilité de la pâte aura permis la mise en forme et en 
acte symboliques du traitement perpétré sur sa peau par une mère absentée, le transfert de l'expérience infantile traumatique dans une forme sensible objectivée.

Le dispositif à médiation artistique "est thérapeutique, soutient Chouvier, dans la mesure où il invite un sujet à adresser quelque chose à un autre qui [...] le transformera psychiquement et l'adressera en retour sur un registre métaphorique et symbolique, par l'entremise du médium malléable» (Chouvier, 2013, p. 341). En d'autres termes, c'est parce que les actes symboliques «sont repris dans le contretransfert qu'ils se réferent à un travail symbolique» (p. 332), contretransfert ou identification projective, ajouterais-je.

Le trauma chez cette patiente n'était certes pas uniquement lié aux épisodes cuticulaires; ceux-ci sont à considérer comme une condensation ou une cristallisation de l'expérience primaire objectale. L'indifférence qu'éprouve l'enfant à son égard par une mère qui n'est pas en mesure de lui renvoyer son propre reflet mais le vide, une mère insaisissable au sens où Roussillon (2013) l'entend, a des conséquences psychiques majeures, dont celle d'empêcher l'illusion d'omnipotence, brimant ainsi la désirance et l'actualisation du potentiel créateur. Quant à l'in-différence qui caractérise toute relation fusionnelle et contraint l'individuation, elle reproduit l'éternel retour du même, l'identique n'ayant lieu que dans la répétition du même, dans ce qui ne fait que se répéter et ne s'élabore pas: pulsionnel de mort psychique, déliaison, comme Freud (1920) nous le donne à penser.

Après des centaines et des centaines de petits objets et de petits animaux, un personnage émergera de la pâte à modeler et prendra une forme humaine, celle d'une femme aux attributs sexuels évidents. Une victoire d'Éros, une "ouverture du sujet à quelque chose [que Rolland] appelle l'espoir» (Rolland, 2006, p. 20).

"L'art invente pour découvrir», soutient Alain Médam (2002, p. 152) en une sorte d'écho aux mots de Paul Klee, bien connus, selon lesquels l'art rend visible. Je ne m'attarderai pas ici à l'œuvre de perte que le prédicat signale et que Pontalis (1986) a bien su faire valoir - si ça rend, c'est que ça a déjà été perdu -, mais dirai en terminant que si l'art invente pour découvrir, c'est qu'il ne connaît pas la vérité qu'il cherche et qui se cherche. Seul l'engagement dans la quête permettra de s'en approcher. La psychothérapie psychanalytique médiatisée serait l'une de ces voies.

Josée Leclerc josee.leclerc@concordia.ca 


\section{Notes}

1. Je reprends ici l'appellation proposée par les analystes lyonnais A. Brun, B. Chavier et R. Roussillon.

2. J'entends le vocable dans le sens générique du terme.

3. Je tiens à dire ce que la présente élaboration doit à la pensée de Jean-Claude Rolland entre autres dans Avant d'être celui qui parle, pour la concordance que j'y trouve avec ma propre théorisation de l'atteinte psychique, tout en reconnaissant que celle-ci n'a pas la même acuité.

4. Je reprends ici les termes de Pontalis et les modifie. Ainsi, à partir de la reprise par Freud dans la Traumdeutung de la métaphore du tisserand chez Goethe, et considérant que «ce que lie le métier à tisser, l'analyse le délie», Pontalis souligne que «les fils entrecroisés conduisent quelque part parce qu'ils viennent de quelque part», à savoir l'ombilic du rêve, «ce cordon originaire qui nous lie à la vie et à la mort» (1978, p. 39).

5. Au sens où Roussillon l'entend, soit le médium malléable «matériel» et la fonction médium malléable du clinicien.

6. La psychothérapie avec cette patiente s'est déroulée en dyade patient-psychothérapeute dans mon cabinet, au sein d'un institut qui offre des services psychothérapiques selon une échelle d'honoraires gradués déterminés en fonction du revenu. Je souligne la différence entre ce dispositif-cadre et celui prévalant en centres de soins psychiatriques; il en est la fonction du tiers: participation de tiers substituables (psychiatre, analyste consultant et analyste traitant, ou équipe traitante), double cadre et modalité groupale sont parmi les modalités d'objectivation inhérentes au travail clinique en centre de soins. - Bien que, dans une moindre mesure, l'institut auquel je suis affiliée agit aussi comme tiers: les patients doivent faire une demande de soin, ils sont contactés par téléphone par un clinicien (non traitant) qui procède à une évaluation psychologique sommaire, et ils doivent venir en personne sur place défrayer le coût de leur inscription avant d'être référés au psychothérapeute traitant.

7. On reconnaitra dans l'invitation à fermer les yeux l'écho de ma propre difficulté à voir ce qu'il me fallait supporter, dans les deux sens du terme.

8. À ses tentatives de relations amoureuses correspondra un nouvel épisode psychotique chez sa mère, signant leur échec.

\section{Références}

Baldacci, J.-L. (2010). Le CCTP dit «Centre Jean-Favreau». Le Coq-héron, 1 (201), 61-67.

Bion, W. R. (2005). Séminaires italiens. Bion à Rome, Paris: Sous presse.

Chouvier, B. (2013). Objet médiateur et groupalité. Dans A. Brun, B. Chouvier et R. Roussillon (dir.), Manuel des médiations thérapeutiques (70-94). Paris: Dunod.

Didi-Huberman, G. (1992). Ce que nous voyons, ce qui nous regarde, Paris: Minuit.

Donnet, J.-L. et Green, A. (1973). L'enfant de ça. Paris: Minuit.

Freud, S. (1920). Au-delà du principe de plaisir. Dans Essais de psychanalyse (45-115). Paris: Payot, 1981.

Freud, S. (1915). Les pulsions et leurs destins. Dans Métapsychologie. Euvres complètes, vol XIII. Paris: PUF, 1988, 161-185.

Freud, S. (1900). L'interprétation des rêves. Paris: Presses universitaires de France, 1987.

Gantheret, F. (1989). L'originaire: la métaphore inaccomplie. Psychanalyse à l'université, 14 (55), 29-48.

Leclerc, J. (2012 a). Quand l'image s'écrit. Une anthologie d'écrits d'artistes sur le thème du dessaisissement créateur. Montréal: Liber. 
Leclerc, J. (2012 b). When the image strikes: Post-modern thinking and epistemology in art therapy. Dans H. Burt (ed.), Art therapy and post modernism: Current trends and new research (367-378). Londres: Jessica Kingsley.

Leclerc, J. (2004). Art et psychanalyse. Pour une pensée de l'atteinte. Montréal: XYZ.

Médam, A. (2002). La tentation de l'œuvre. Montréal: Liber.

Pontalis, J.-B. (1988). Perdre de vue. Paris: Gallimard.

Pontalis, J.-B. (1986). La jeune fille, préface de Le délire et les rêves dans la Gradiva de Jensen. Paris: Gallimard.

Pontalis, J.-B. (1978). Les vases non communicants. Nouvelle revue française, 302, p. 26-45.

Rancière, J. (2003). Le destin des images. Paris: La Fabrique éditions.

Racker, H. (1963). Transference and Countertransference. New York: Inter University Press.

Rolland, J-C. (2006). Avant d'être celui qui parle. Paris: Gallimard.

Roussillon. R. (2013). La fonction médium malléable et les pathologies du narcissisme. Dans A. Brun, B. Chouvier et R. Roussillon (dir.), Manuel des médiations thérapeutiques (p. 188-202). Paris: Dunod.

Roussillon. R. (2010). Propositions pour une théorie des dispositifs thérapeutiques à médiations. Le Carnet PSY, 1 (141), 28-31.

Schaverien, J. (1992). The revealing image. Londres: Routledge.

Weir, F. (1987). The role of symbolic expression in its relation to art therapy: Kleinian approach. Dans T. Dalley (dir.), Images of art therapy: New developments in theory and practice. Londres: Tavistock.

Winnicott, D. W. (1951). De la pédiatrie à la psychanalyse. Paris: Payot, 1989. 